\title{
La Política Exterior de Cuba en el Sistema Moderno de las Relaciones Internacionales
}

\author{
Cuba's foreign policy in the modern \\ system of international relations
}

\author{
Helen Sugelly León Ortega
}

\section{Resumen}

La República de Cuba es un Estado socialista en el hemisferio occidental, cuyo sistema fue establecido como resultado de la victoria del movimiento revolucionario en 1959, en el marco de un sistema post-bipolar que ha sufrido muchos cambios. El presente texto pretende analizar el estado actual de la política exterior de la Isla. Con ese objetivo y con la ayuda de la bibliografía científica compendiada, se establece que, hasta el momento, con la figura de Miguel Mario Díaz-Canel Bermúdez en el poder, hay muestras de lo que sería el perfeccionamiento del socialismo. El cual viene dado por un nuevo modelo de relaciones internacionales, diseñado principalmente en torno a EE. UU., China y Rusia: el primero como su enemigo comercial y los dos últimos como sus aliados.

Palabras clave: Relaciones internacionales, Sistema Post-bipolar, Política exterior.

\section{Abstract}

The Republic of Cuba is a socialist State in the western hemisphere, its system was established as a result of the victory of the revolutionary movement in 1959, but in a Post-bipolar System it has undergone many changes. The present text tries to analyze the current state of the Island's foreign policy. With that objective and with the help of a summarized scientific bibliography, it is established that, up to now, with Miguel Mario Díaz-Canel Bermúdez in power, there are samples of what would be the perfecting of socialism, marked by a new inclination of international relations, designed mainly around the USA, China and Russia, the first as its commercial enemy and the last two as its allies.

Keywords: International relations, Post-bipolar system, Foreign policy.

\section{Introducción}

El presente texto es el resultado de una revisión bibliográfica en torno a las estrategias de política exterior de Cuba para el desarrollo del modelo post-bipolar y la transición a un mundo multipolar. Es así que, las relaciones internacionales y la política exterior se convierten en un análisis particularmente relevante del papel y del lugar de los Estados que se desarrollaron como parte del sistema moderno, cuanto más, dadas sus condiciones de evolución constante. Ejemplo de ello es la República de Cuba. El cual es un Estado Socialista que estableció su Sistema en la década de 1960, bajo el liderazgo de F. Castro. Ya para noviembre de 2016, tras la muerte de este último, R. Castro asume el compromiso de "defender, mantener y continuar perfeccionando el socialismo", en sus propias palabras. Y en abril de 2018, Miguel Mario Díaz-Canel Bermúdez, asume el cargo de primer dirigente, comenzan- do lo que muchos llaman, una nueva era de continuidad, con algunas señales de modernización.

Para el caso de los factores externos de la Política, estos incluyen, por una parte, el mandato presidencial de Donald Trump en EE. UU., fundamentalmente diferente de la administración de Obama respecto al restablecimiento de relaciones con la Isla. Por otro lado, su constante aliado, Venezuela, ya no puede proporcionar a Cuba todo lo que suministró hasta hace unos años. Por lo que, en la búsqueda de nuevos inversores, ha establecido fuertes lazos con China y Rusia.

Entre tanto, la atención se centró en el devenir histórico de las relaciones internacionales de Cuba, intentando estructurar las circunstancias en que las mismas se han forjado a partir de los hechos internacionalmente públicos y las interacciones en estos. Haciendo un llamado 
especial al trabajo de Rosenau, con las variables más importantes dentro del análisis de la política exterior y para su entrega final, el texto fue estructurado en cuatro apartados así:

1. Marco analítico del asunto;

2. Cuba después del colapso de la URSS;

3. Cuba y sus aliados internacionales;

4. Cuba en el escenario mundial 2016-2025.

\section{Marco Analítico del Asunto}

El análisis de la política exterior, permite comprender mejor cómo los actores políticos toman decisiones al momento de relacionarse con entidades extranjeras y, dada su variabilidad, es útil contar con modelos de procesos analíticos que ayuden a explicar las razones por las cuales los Estados actúan. En primer lugar, corresponde aclarar que la política exterior y la diplomacia son dos conceptos fundamentalmente diferentes, y lo importante acá es que mientras la primera traza las directrices de la acción internacional de cada Estado, la segunda se ocupa de su ejecución. En este contexto, una y otra están íntimamente relacionadas.

Según León (2012), los estudios de política exterior, en la mayoría de los casos, se basan en métodos de investigación empírica para identificar las características distintivas, las similitudes y las diferencias entre los Estados. Algunos autores emplean el análisis de los antecedentes históricos, en cambio, otros, presentan aspectos teóricos para examinar cómo las circunstancias afectan la política exterior. Entre tanto, para el presente ejercicio, se hará uso del trabajo de Rosenau, analizando variables de política exterior de la categoría de las idiosincrásicas, las funcionales, las burocráticas, las nacionales y del contexto internacional (Izuel et al., 2015; Santa Cruz, 2014).

Por supuesto, el espacio limitado en esta oportunidad, hará que algunos temas sean abordados superficialmente, solo con el ánimo de traerles a colación para que hagan parte del análisis, pero, sobre todo, teniendo como fin el estudio de los intereses relacionados con épocas históricas, liderazgo político, alianzas, balance de poder, relaciones comerciales, servicio diplomático profesional y otros aspectos. Mientras tanto, se sintetizarán los temas más coyunturales de la Isla, para ir construyendo el análisis.

\section{El éxodo del Mariel - Idiosincrasia de Fidel.}

Para hablar un poco de variables idiosincrásicas del liderazgo cubano, parece oportuno hacer uso de uno de los episodios más sensibles de la Isla. Y es que, pocos experimentos han obtenido la amplitud de cobertura como la diáspora de aproximadamente 120,000 cubanos saliendo desde el puerto de Mariel hasta Miami, entre abril y septiembre de 1980. Que por los demás, generó un mar de posibilidades y decisiones que tuvieron un fuerte legado (Sanabia, 2019).

Para el caso, en abril de 1980, F. Castro anunció la apertura del Puerto de Mariel a cualquier cubano en la Isla que quisiera irse. Los cubanoamericanos en Florida inmediatamente movilizaron botes al puerto de Mariel para recoger a sus familiares. La Casa Blanca asumió la responsabilidad de tratar de controlar la crisis y se adquirió una posición única, con un espectro completo de posibilidades: desde la designación general de refugiados hasta la intervención militar y la obstrucción de los botes. Para Panitch y Gindin (2015), la inesperada magnitud de la crisis, hizo que la implementación de políticas se viera limitada por el desorden organizacional y las agendas burocráticas conflictivas. Si bien el presidente Carter tenía una fuerte afinidad por los Derechos Humanos, el inconveniente era que prácticamente el gobierno cubano dictaría la política y EE. UU. tendría que aceptar tantos inmigrantes como Castro quisiera enviar.

Es así como este ejercicio de coerción, desde la perspectiva de Castro, demostró ser un éxito calificado: se trataba del pueblo cubano como arma política asimétrica contra EE. UU. Para Gómez (2015), el éxito de Castro se basó en su capacidad para internacionalizar su propia crisis doméstica y transformarla en una crisis tanto de política exterior como de política estadounidense. Aun cuando, como señala Alzugaray (2014), el resultado de esto excedió a las intenciones primarias, lo importante aquí es corroborar como una acción puede modificar el rumbo habitual de política exterior y de relaciones internacionales de un país. Más adelante se hablará un poco más, acerca de las herramientas que aun con el reproche internacional, todavía son empleadas por Cuba. 


\section{Cuba, Después del Colapso de la URSS}

A finales de la década de 1980, y especialmente después del colapso de la Unión de Repúblicas Socialistas Soviéticas URSS, Cuba enfrentaba serios desafíos tanto a nivel interno como externo. Privada del apoyo económico de su gran aliado, la economía colapsó y debido a la crisis en la industria azucarera, no pudo cumplir los acuerdos con Moscú sobre el programa de petróleo por azúcar y, eso le llevó a una escasez significativa de recursos energéticos. Refiere Arias Castaño (2016) que, Cuba sufría una insuficiencia de bienes esenciales y la proclamación de un "Período especial en tiempo de paz", que en la práctica significaba un régimen de ahorro máximo en absolutamente todo.

Las dificultades económicas obligaron al Gobierno a emprender reformas de mercado. En 1992, se adoptaron una serie de enmiendas constitucionales que permitieron el desarrollo del turismo y simplificaron el desarrollo de los negocios extranjeros en la Isla. F. Castro expresaba su intención de defender el socialismo y de mantener un monopolio estatal en las áreas más importantes de la economía. Pero, como se verá más adelante, desde principios del siglo XXI, la política exterior de Cuba empezó a mostrar un papel activo en las relaciones internacionales (Hernández et al., 2013; Chaveco y del Río, 2010).

Su cercanía geográfica con EE. UU. y el papel geopolítico de Washington fueron las primeras claves de la renaciente política exterior. En 2008, Obama promovió la liberalización de la posición de este país en relación con Cuba, pero era aún temprano para hablar sobre la normalización absoluta de las relaciones (Márquez Covarrubias, 2010; Serbin, 2016). Banderas Bustillos (2016) resalta que, desde la crisis caribeña, el Gobierno cubano se percató no solo de la inconveniencia de usar armas atómicas como un medio protección, sino también del hecho de que la existencia de estas armas en el país, podría convertirse en una base real para la intervención de Washington.

El colapso de la URSS marcó el comienzo de un deterioro en la relación entre Cuba y su sucesor, pues los líderes cubanos decidieron construir una política exterior hacia varios actores de las relaciones internacionales, entre otras cosas, para establecer fuertes lazos con países de ALC (Vélez Pretelt, 2011; Domínguez Guadarra- ma, 2015). Y cabe destacar que, la mayoría de los países miembros de la Organización de las Naciones Unidas apoyan a Cuba para levantar el embargo de EE. UU., ejemplo por supuesto, de su buena diplomacia actual. Campos (2011) señala que, en la misma línea, el Gobierno cubano también sentó una dura crítica a la Resolución del Consejo de Seguridad, que sirvió de base legal para las operaciones militares contra el Gobierno de Libia y en un momento condenó las sanciones de la ONU contra Irán.

Así mismo, indica Malamud (2011), todo esto en general se ajusta a la estrategia de la diplomacia cubana orientada al apoyo a los países del Tercer Mundo que están bajo la presión de Estados desarrollados. Un concepto de política exterior construido a través del realismo clásico, donde el papel dominante es jugado por los intereses nacionales y la ganancia personal en los procesos políticos en el mundo. Conforme exponen Alburquerque y Alvarado (2019), Cuba no deja de promover activamente el avivamiento del Movimiento de los No Alineados, con la esperanza de usarlo como un nuevo "polo" en la construcción de un mundo multipolar.

\section{Cuba en el Sistema Moderno de Relaciones Internacionales}

El actual sistema internacional, emergió en 1991 después del colapso de la URSS. Este sistema más conocido como el "Sistema Postbipolar", se caracterizó por el hecho de que no se formó como resultado de un tratado de paz y fue más bien un período histórico de cambios (Iglesias, 2002; i Lecha, 2011). Sobre todo caracterizado por la variabilidad y un alto grado de inestabilidad. De igual forma, la fase final de la formación de la post-bipolaridad (2010 2014), estuvo marcada por la falta de un claro centro de poder, en que el derecho a entrar en el núcleo del sistema político internacional fue disputado por 10-15 Estados que estaban dispuestos a hacer lo que fuera necesario por participar activamente en la restructuración de una institucionalidad en revaluación y con tendencias que desafiaban el orden anterior (Hornero, 2016; Sarsenov y Raev, 2018).

Por supuesto, esta restructuración, exigió a todos los países revaluarse y evolucionar en tanto fuera posible. El proceso de Cuba, en especial, comenzó en 2008 con el retiro de F. Castro y 
la transferencia de autoridad a R. Castro. Al principio, todo parecía permanecer sin cambios, pero, pronto comenzaron a darse pasos de progreso económico, en lo que al interior del gobierno se conoce como el "perfeccionamiento del modelo".

Económicamente, el paso más importante fue la Ley de 2011 sobre inversión extranjera y a propósito de una zona especial de desarrollo alrededor del puerto de Mariel. Dice Berg-Rodríguez (2019) que, su tarea era atraer capital extranjero para el desarrollo de la economía nacional. Empresarios de México, Brasil y otros países latinoamericanos, se apresuraron en llegar. Rusia aseguró su lugar en proyectos conjuntos de inversión en la Isla, condonando el $90 \%$ de su deuda con Moscú. Y en enero de 2016, se estrecharon relaciones con el presidente francés, Francois Hollande, quien expresó interés en profundizar tanto la cooperación bilateral, como aquella con los países de la UE (Duany et al., 2019).

Políticamente, también se produjeron cambios importantes. Quizá el más importante de ellos, según refiere Rodrigo (2016), fue que el Congreso propuso el reajuste de la economía, como una de las prioridades nacionales. Así que, aunque el Gobierno era enfático en que el mismo debía ser lento para asegurar la inviolabilidad del sistema político y social, con todo, los cambios han asegurado la irreversibilidad del proceso que condujo a movimientos importantes en la Sociedad.

En todo caso, aun sin cambios internos radicales, Cuba está pasando por un período de brillantes victorias diplomáticas que era difícil imaginar después de décadas de aislamiento. Es así que, el Gobierno atrae empresarios e inversores de todo el mundo para participar en el desarrollo de un país con una posición geográfica favorable y con mucha fuerza laboral. La economía, para Silva (2014), se está reconstruyendo actualmente en el sector terciario: los servicios representan una gran participación en la estructura de las exportaciones cubanas, y mientras que el aporte de los productos básicos está disminuyendo, el turismo refleja su mejor época.

Con ocasión de esto último, uno de los mayores logros ha sido la construcción del Puerto Mariel que busca convertirse en una herramienta para aumentar el comercio exterior, presentándose como un puerto único en ALC. Para Flores (2018), el Gobierno cubano está tratando de seguir, en fin, un camino similar al que China ha elegido, manejando buenas relaciones comerciales y financieras con el mundo, independientemente de las diferencias existentes por ejemplo en el campo de los Derechos Humanos.

Además de ello, Cuba se ayuda con la criticada y elogiada diplomacia médica, de la cual se ocupa hace varias décadas (Duany et al., 2019). Perea Ozerin (2014) indica que, Cuba comenzó a usar sus médicos como una herramienta diplomática para superar el aislamiento político en 1963, un año después de ser expulsada de la OEA. Envió su primera misión médica a Argelia, con 56 cubanos reemplazando a médicos franceses que abandonaron el país africano después de que se independizó de Francia en 1962. Desde entonces, comenzó a ganar bonos políticos, pues, los médicos cubanos en el extranjero ayudan a crear un "gesto amable" para el régimen autoritario.

Respecto a sus puntos restrictivos, reseña Perea Ozerin (2014), La Habana entiende que una misión extranjera está cargada de riesgos que pondrían en peligro tanto las actitudes ideológicas como la reposición del tesoro nacional (suponiendo que uno de los médicos decida no regresar a la Isla); por lo cual, el Gobierno ha desarrollado un código especial que les prohíbe entablar relaciones matrimoniales con ciudadanos de los países en los que trabajan y les ordena comportarse de acuerdo con los ideales de la Revolución (Sanabia, 2019).

Sus fundamentos fueron establecidos por F. Castro (Serbin, 2011) y han convertido a la diplomacia médica en una herramienta ideológica, diseñada para influir en la imagen externa del país (Vélez Pretelt, 2011). La línea estratégica del gobierno, es mejorar su economía con la ayuda de las divisas y la misión de los médicos es difundir la idea de que un modelo autoritario, puede salvar vidas.

Y a juzgar por los resultados, es una de sus mejores políticas, aun cuando en los últimos años, la crisis en Venezuela y el cambio de poder en Brasil (los dos Estados que representan la mayor parte del personal médico cubano), amenazaron con colapsar esta fuente de ingresos tan importante para Cuba (Sanabia, 2019). Por lo que, sus misiones médicas a Italia y Andorra (con ocasión de la pandemia), donde la situación política es mucho más estable que en América Latina, son, si no una salida, un punto importante para 
resolver los problemas financieros cubanos. No es tabaco, ni azúcar, pero los médicos siguen siendo los productos de exportación más rentables para Cuba.

\section{Cuba y sus Aliados Internacionales}

La política exterior que hoy se observa, se forjó con el transcurso de muchos años de lucha contra todo tipo de sanciones de Washington, contra el agresivo bloqueo comercial, económico y financiero $\mathrm{y}$, contra los constantes intentos de aislar a Cuba, sofocando su actividad sociopolítica. Y hoy, la diplomacia cubana continúa trabajando activamente en todos los ámbitos de la vida internacional, buscando lograr un mundo seguro y una cooperación igualitaria con todos los Estados, pero como en todos los procesos, se muestran algunas inclinaciones que se analizarán a continuación.

\section{Integración latinoamericana y caribeña: oportunidades y desafíos}

La historia de las organizaciones intergubernamentales de América Latina y el Caribe ALC puede explicar su evolución en dos fases: la primera tuvo vigencia entre los años sesenta y setenta, bajo la inspiración del regionalismo del desarrollo irradiado por la CEPAL. La segunda etapa, llamada regionalismo neoliberal, comienza con las transiciones democráticas de la década de 1980 y corresponde a la prescripción del Consenso de Washington al ámbito de las políticas económicas regionales.

A este esquema, podría agregarse una tercera fase, caracterizada por la desviación del paradigma integracionista anterior, que correspondería a la creación de ALBA y CELAC y que tocan de cerca a Cuba. En cualquier caso, dice Llenderrozas (2013), el regreso a la cuestión de la autonomía demuestra que la región de ALC del siglo XXI sigue enfrentando el hecho de que no ha logrado implementar cambios estructurales en la esfera interna y todavía está influenciada por poderes "extrarregionales". De ahí la importancia de reflexionar críticamente sobre el papel de estas experiencias.

Y es que, a pesar del surgimiento del regionalismo autónomo, las condiciones de la periferia latinoamericana (caracterizadas por un legado histórico de fragmentación intrarregional y un pasado colonial que transformó a la región en un proveedor de materias primas), nunca han desaparecido, en comparación con un centro con capacidad de innovación tecnológica en sectores clave (Caetano y Sanahuja, 2019). Y es que, como indica Flores (2018), los cambios políticos experimentados recientemente por diferentes países de la Región, combinados con la estructura eminentemente intergubernamental de los organismos regionales en general, ya sean autónomos o subordinados, han puesto de relieve la cuestión de suficiencia de las reformas políticas para conducir una reconfiguración de la integración.

Y sobre todo, impone el cuestionamiento de si Cuba podrá mantener políticas regionales sobre problemas comunes que se probaron a través de ALBA y CELAC, además de enfrentar el desafío de consolidar las agendas sociales, productivas y ciudadanas que surgieron durante este período (Caetano y Sanahuja, 2019). Por supuesto, refieren Kuwayama y Rosales (2012), no puede obviarse que una de las principales características positivas de CELAC tiene que ver con su construcción, basada en la unidad de ALC, como actor internacional, concretada, por ejemplo, a través del diálogo multilateral obtenido con China y la Unión Europea. CELAC representa una novedad en el escenario regional tradicional, al vincular disputas para un modelo de desarrollo con cuestiones de dimensión social, derechos regionales, enfoque territorial, sociedad civil e identidad. Su futuro, sin embargo, depende de diferentes variables y posibles escenarios para combinar las fortalezas de los actores políticos y sociales involucrados.

ALBA, por su parte, se define como una plataforma de integración cuyo propósito fundamental es lograr el desarrollo integral para ALC a través de un proceso de integración inspirado en Simón Bolívar y José Martí. Al contar con la unión de los regímenes izquierdistas del continente, pronto comenzó a tener un impacto significativo en la situación geopolítica en el hemisferio occidental (Caetano y Sanahuja, 2019). Han celebrado extensas cumbres, firmado cientos de declaraciones y acuerdos conjuntos, formado consejos políticos, económicos y sociales, e implementado proyectos en áreas clave de cooperación. Cuentan con el aval de Rusia y el análisis de las formas de interacción dentro del marco de esta asociación permite establecer cierta analogía con el Consejo de Ayuda Mutua 
Económica, que una vez existió en las extensiones del antiguo campo socialista (Serbin, 2016).

En la actualidad, la alianza está experimentando dificultades económicas y políticas. Las razones económicas se deben a la caída en los precios del petróleo y a la incapacidad de mantener aliados en tal volumen (Sanabia, 2019). Las dificultades políticas se expresan, en primer lugar, en que bajo el mandato de Lenin Moreno, Ecuador abandonara la alianza. Y después que, junto con la destitución del poder del presidente Evo Morales, Bolivia también lo hiciera. Por otro lado, como exponen Duany, et al. (2019), el presidente de Nicaragua, Daniel Ortega, después de los acontecimientos de abril de 2018 relacionados con el intento de llevar a cabo la reforma pensional, se tambaleó gravemente y ha convertido al país en un punto caliente en la región.

La alianza está perdiendo su posición anterior en el continente. Pero vale la pena tener en cuenta el efecto demostrativo del impacto en las masas, ya que muchos de sus proyectos son muy atractivos para ellos. Al mismo tiempo, no se debe sobrestimar su importancia para predecir las perspectivas de desarrollo político y especialmente económico de la región, aun cuando la alianza toma una trayectoria extraña y hoy sus perspectivas son extremadamente nebulosas.

\section{La República de Cuba y los países de las Américas}

Durante por lo menos, medio siglo después de la victoria de la Revolución Cubana de 1959, el Gobierno de EE. UU. trató de aislar a la Nación rebelde. Sin embargo, a pesar de todos los esfuerzos realizados por la superpotencia mundial, el objetivo no se logró del todo, pues el actual Régimen ha sobrevivido al aislamiento de diez administraciones presidenciales estadounidenses, sin que esto implique no tener otras relaciones internacionales. Lo que si es cierto es que las relaciones con Washington tradicionalmente juegan un papel crucial en la agenda de la diplomacia cubana, después de que en enero de 1961 el presidente Dwight Eisenhower rompiera relaciones con Cuba (Ayerbe, 2016; Cavicchioli, 2014).

En las últimas décadas, para Edwards (2015), uno de los puntos fuertes de la diplomacia cubana, ha sido la exclusión de la Isla en la lista de Estados que apoyan el terrorismo. Los americanos notan que el país se encuentra es un "estado de transición" y que no es un peligro para la Seguridad Nacional. Así que, incluso si el embargo estadounidense se mantiene, Cuba seguirá siendo atractivo para la inversión de los países occidentales.

Por su parte, la restauración de las relaciones con EE. UU. en 2014 dio una esperanza para que La Habana acelerara tanto en la reforma económica como en la liberalización política, cuando los entonces presidentes de EE. UU. y Cuba, anunciaron el acercamiento entre los gobiernos de ambos países, tras más de cincuenta años de alejamiento (Feinberg, 2011; Zovatto, 2014). Los dos primeros años resultaron positivos, marcados por las facilidades de viaje y de comercio entre los países vecinos. Sin embargo, al llegar Donald Trump a la Casa Blanca, la relación reanudó viejas tensiones y añadió algunas nuevas.

Durante los últimos tres años ha habido una erosión progresiva del descontento de EE. UU. hacia Cuba, alegando violaciones a los Derechos Humanos y apoyo a la dictadura venezolana. Por ello, la política exterior cubana exhibe también especial prioridad hacia América Latina y de hecho, el relativamente reciente "giro a la izquierda" en América Latina ha llevado, a fortalecer la cooperación entre Cuba y los países de la Región (Stoessel, 2014). Además, La Habana se unió a la Asociación Latinoamericana de Integración ALADI y firmó acuerdos comerciales preferenciales con todos sus participantes y con los países del Mercado Común del Sur MERCOSUR.

Y aun con la difícil situación de Venezuela, vale anotar la existencia de una zona de libre comercio y una cooperación activa en el sector de la salud y la educación. Por otro lado, y como parte de los programas de seguridad económica de Cuba, ayuda a Ecuador, Bolivia y Nicaragua a desarrollar sistemas salud y educación, y en un pasado reciente también contaba con una amplia política de diálogo con países influyentes de la región, incluido Brasil, Argentina y Chile (Natanson, 2012).

\section{Cooperación con la República China}

Las relaciones chino-cubanas tienen ya muchos años de mutuo beneficio. El punto de referencia para la relación entre los dos Estados, 
es el 28 de septiembre de 1960. Ríos (2016) relata que, Cuba se convirtió en el primer país de Latinoamérica que reconoció la República Popular de China y que estableció relaciones diplomáticas con ella. China respaldó solidariamente la Revolución Cubana. Por ello, Cuba mostró especial diligencia en la adopción de medidas para desarrollar las relaciones entre ambas naciones. El 1 de septiembre de 1960 en la Primera Declaración de la Habana, F. Castro anunció el rompimiento de relaciones con Taiwan, para establecerlas con China. Y el 28 de septiembre del mismo año, se lanzó un Comunicado Conjunto sobre el Establecimiento de Relaciones Diplomáticas.

Sin embargo, durante los años sesenta y principios de los setenta, China tenía poco que ofrecer en términos de asistencia económica, y la relación se complicó por la división chino-soviética. La dinámica de las relaciones chino-cubanas cambió cuando la URSS se derrumbó en 1992. Cuba se encontró económicamente vulnerable cuando la generosidad soviética terminó rápidamente (Santos Brito, 2019). La década de 1990, detalla Fazio Vengoa (2012), hundió a Cuba en una depresión económica prolongada, marcada por la escasez crítica de petróleo y una amplia gama de productos básicos. Las condiciones eran tan malas que aproximadamente 30,000 cubanos huyeron de su país. Y China, ahora una historia de éxito económico y activa en los asuntos mundiales, se volvió más atractiva para Cuba, que se vio obligada a buscar nuevos socios comerciales y de inversión.

Quizá su mayor auge en la actualidad se debe a la crisis económica de Venezuela que provocó un fuerte impacto en el intercambio comercial de la Isla. Desde entonces, el diálogo interestatal con China ha sido probado en busca de fuerza, los países encontraron un terreno común y han permanecido activos en el desarrollo de la cooperación y, no puede esconderse la base para su acercamiento político (Duany et al., 2019). Primero, ambos han elegido como modelo para su desarrollo al Socialismo. Aunque, por supuesto, el socialismo de Cuba es diferente al socialismo de China. Fazio Vengoa (2012) describe: El primero se basa en gran medida en el soviético y requiere algo de modernización y, el segundo representa una simbiosis de una economía centralizada, adaptando el marxismo-leninismo a las circunstancias chinas. En segundo lugar, las po- siciones de las partes en asuntos internacionales son a menudo similares.

Beijing ha expresado su apoyo para levantar el bloqueo económico de Cuba y su regreso del aislamiento internacional al Sistema Mundial de derechos como miembro pleno. Después de la muerte del autor intelectual de la Revolución Cubana, no se apagó el camino socialista y sí se aceptó una versión china de desarrollo. En varias reuniones, puntualiza Alzugaray (2014), las partes han reafirmado su compromiso con los valores comunes y han discutido las perspectivas bilaterales, con la intensificación de la cooperación en el campo de la educación y las tecnologías de información, basándose en cuatro principios (Duany et al., 2019): el desarrollo de industrias, el comercio abierto y el desarrollo cultural e intelectual de los países (por el cual ambas partes deben compartir su experiencia en el desarrollo de nuevas tecnologías y colaborar en el uso y desarrollo del potencial intelectual).

En el 2014 se firmaron 29 nuevos acuerdos, cuyo contenido se refería a diversas áreas, entre las que destacan las finanzas, la biotecnología, la agricultura, la infraestructura y las fuentes de energía renovables (Serbin, 2015). Además, China ha aprobado diversos préstamos para la modernización de la Isla y a pesar del pequeño número de empresas comunes, el país asiático tiene la intención de invertir en proyectos de turismo y agricultura cubana (Duany et al., 2019). Y definitivamente una gran ventaja en las relaciones bilaterales es que, China invierte enormes fondos en producción de níquel.

Caetano y Sanahuja (2019), señalan también, los múltiples beneficios que tendría para China un levantamiento del embargo de EE. UU. a Cuba. Tan solo una relajación de sanciones y un levantamiento parcial del embargo sentaría las bases para un número ilimitado de proyectos de empresas chinas en Cuba. La Isla tiene una ubicación importante, porque está en el cruce de América del Norte, Central y del Sur, además tiene los cinco mejores puertos del Caribe, siendo estos los mayores llamativos para el Gobierno chino. A diferencia de EE. UU., China, busca no perder los resultados logrados en la dirección cubana y muy probablemente, continuará aumentando la cooperación, pues Cuba es una pieza clave para la inversión con el fin de convertirla en una especie de centro logístico. China, 
expone Gómez (2013), utiliza con gran éxito los mecanismos de poder blando en su estrategia de política exterior y Cuba, ha tenido una tendencia positiva con su ejemplo.

Mientras tanto China y EE. UU. se consideran rivales clave para el título de líder global del siglo XXI. El resultado de su confrontación establecerá una nueva alineación en el tablero de ajedrez mundial. En este sentido la competencia de los dos gigantes ocurrirá en todas las regiones de la tierra y por lo pronto, uno de los mayores logros de China en América Latina es la cooperación intensiva con la CELAC, pues incluye 33 Estados de la Región (Correa Serrano y Catalán Salgado, 2016). Y al final, muchos detractores cubanos señalan que aun cuando Cuba declaró que conduciría la política exterior sin depender de ningún actor externo, la experiencia refleja lo contrario, pues la economía cubana se mantiene a flote gracias al mérito de China. Para 2018, China había asumido un papel estratégico importante en la economía cubana, del cual el liderazgo del estado insular es muy consciente (Caetano y Sanahuja, 2019).

Cuba finalmente está experimentando un cambio de guardia política, mientras pasa de R. Castro a Miguel Díaz-Canel, la economía cubana está nuevamente en pie de lucha y las condiciones externas se han vuelto más difíciles. El liderazgo de Cuba tendrá que tomar decisiones difíciles en la era posterior a Castro y, tanto los problemas como su resolución apuntan a la relación chino-cubana. En muchos aspectos, Cuba enfrenta los mismos desafíos que enfrentó China en la década de 1970, cuando el asiático se embarcó en su propia reforma bajo la guía de Deng Xiaoping (Zúñiga, 2012). En el caso de China, las limitaciones de planificación central, los principales problemas de producción, los cuellos de botella de distribución y la corrupción empujaron al Gobierno a introducir reformas de mercado. En cierto sentido, para Caetano y Sanahuja (2019), China pasó de estar atada ideológicamente a una menor rigidez ideológica y a adoptar más el pragmatismo y esta es la lección crítica que el nuevo liderazgo cubano debe aprender.

A China le interesa ver una Cuba exitosa bajo el Partido Comunista (Sanabia, 2019). En el juego más amplio de la geopolítica mundial, tener una Cuba pro China, con su ubicación estratégica justo al sur de EE. UU. y a horcajadas en las principales rutas marítimas, tiene mucho sentido. Teniendo en cuenta que EE. UU. desafía el dominio chino en el mar de China Meridional, China puede desafiar el dominio estadounidense en el Caribe en su propio "patio trasero" (Alburquerque y Alvarado, 2019). En muchos aspectos, China está desempeñando un papel similar al de la URSS durante la Guerra Fría, aunque el objetivo principal del compromiso del país asiático hasta ahora es económico más que militar.

Cuba enfrenta desafíos sustanciales en lo que respecta a la era posterior a Castro. La economía no funciona, la población está inquieta, las relaciones con EE. UU. siguen siendo complicadas y nadie está dispuesto a intensificar y brindar el mismo nivel de asistencia que Venezuela. En esta atmósfera de incertidumbre, Cuba ve a China como un aliado revolucionario y como un importante partidario económico del Régimen. Por su parte, China considera a Cuba como un aliado con una excelente ubicación geoestratégica y proporcionará una buena cantidad de asistencia. Pero es probable que Beijing no ponga en peligro el comercio estadounidense (mucho más importante), al convertir a la Isla en un nuevo puesto avanzado de la Guerra Fría. El mejor aliado de China es aquel con el que puede comerciar y compartir prioridades geopolíticas, asî como uno que se mantenga en pie económicamente, algo que Cuba aún necesita hacer, para que la relación chino-cubana dé el siguiente paso.

\section{Cooperación con la Federación Rusa}

Los últimos años han sido testigos de intercambios de alto nivel sobre una cooperación económica y política más profunda y una intención de defender el derecho de Cuba a elegir su propio futuro, por parte de Rusia, sobre todo, en lo relacionado a la presión estadounidense. Rusia promueve el apoyo al pueblo y a los líderes cubanos, no solo a través de métodos morales o políticos, sino también a través del desarrollo de proyectos de cooperación, comercio y economía que contribuyan a hacer que la economía de la isla sea más sostenible frente a los problemas externos.

Mucho se habla de que la mejoría en la dinámica del desarrollo económico de Cuba está diseñada para convertirse en el vector ruso de su política mundial. En este sentido, es extremadamente relevante el establecimiento 
de medidas mutuamente beneficiosas en la Cooperación ruso-cubana en diversos campos (Rodríguez, 2011; Velez Vargas, 2015). En los años 90, las relaciones se suspendieron debido al colapso de la URSS y, sin embargo, en 1996, se produjo la Declaración sobre principios de las relaciones entre la Federación de Rusia y Cuba, que señaló centrarse en la lucha conjunta contra el tráfico de drogas, el crimen organizado, el terrorismo internacional y el contrabando de armas. Destacando que desde el 2005 Rusia se ha recuperado y está notablemente intensificada (Santos Brito, 2019).

La declaración se convirtió en la base de las relaciones ruso-cubanas modernas y dio lugar a una nueva etapa de interacciones. La actitud de Cuba hacia las amenazas contemporáneas emergentes y la alineación actual de fuerzas coincide en gran medida con la posición rusa, motivo para profundizar las relaciones. Menciona Bueno (2015), Cuba como Rusia, representan un mundo multipolar y la presencia de varias fuerzas en las Relaciones Internacionales. Cuba y Rusia tienen principios compatibles de no injerencia en los asuntos de otros Estados e igualdad soberana y son países que renuncian al uso de la fuerza contra la integridad territorial de los Estados (Santos Brito, 2019).

Entre los ministros de relaciones exteriores de los dos Estados, se ha establecido un diálogo político: las partes cooperan constantemente en la Asamblea General de la ONU y celebran reuniones oficiales. Cuba está colaborando con Rusia en áreas como la ecología, la salud y la cultura. En 2009, alude Serbin (2011), se firmó un acuerdo por un período de 25 años para mantener la exploración y producción de hidrocarburos en la Isla, y Rusia también restableció la cooperación con Cuba en la industria del níquel: en 2008 se firmó un memorando sobre la cooperación en la exploración de depósitos en las minas de Nicaro. Entonces Cuba, en colaboración con Rusia, presta también su apoyo a nivel internacional.

En cuanto a los requisitos para los inversionistas extranjeros, Cuba exige que las inversiones deben ser en forma de creación empresas conjuntas donde la participación del lado cubano debe prevalecer, de esa forma el Gobierno cubano está intentando asegurar la posición del Estado, con una apertura gradual del país (Jeifets y Jeifets, 2017). Rusia invierte en programas de reconstrucción de infraestructura de transpor- te, suministro de agua y producción agrícola; modernización de los sistemas de energía, gasificación de asentamientos; y construcción de infraestructura turística. En 2010, Rusia colaboró activamente con Cuba en áreas como la agricultura y la economía. Y vale la pena considerar que, cuando Rusia ha dejado de cooperar con Cuba, esencialmente ha perdido, porque las fábricas y plantas han traído buenas ganancias a ambas partes (López Zea y Zea Prado, 2010).

Entonces, la política exterior hacia Rusia es eficaz. Se ha creado una base legal para la Cooperación beneficiosa en ambos países. Cuba ve a Rusia como uno de los socios más importantes en el ámbito internacional, y comparte sus puntos de vista en muchos de los problemas mundiales. Cuba puede usar el ejemplo de Rusia porque la reestructuración de su régimen actual conserva elementos del pasado comunista soviético. Y desde un punto de vista económico, la cooperación con Rusia también es beneficiosa para Cuba, pues se encuentra entre sus cinco principales socios operacionales, explorando incluso esquemas económicos más eficientes para la colaboración, sugiriendo negociaciones en una moneda distinta al dólar estadounidense, aun cuando no tiene la fuerza financiera de China o EE. UU.

Las sanciones unilaterales de EE. UU. ofrecen a Rusia, oportunidades geopolíticas y económicas en las Américas y, en contraste con su respuesta en Venezuela, dan como resultado que Moscú desee participar activamente y ayudar a Cuba a asegurar su futuro (Bobes et al., 2015). Empero, antes de alabar por completo la presencia de Rusia, deberá demostrarse que su interés es más que un oportunismo geopolíticamente similar a la visión del mundo transaccional del presidente Trump. En todo caso, a medida que cierran los corredores del dólar estadounidense, La Habana se aleja del abastecimiento de compañías occidentales $y$, se abraza a una relación con Rusia, no solo como un socio que entiende y conoce, sino que ofrece plazo, fiabilidad, estabilidad y soporte tecnológico (Santos Brito, 2019).

\section{Cuba en el Escenario Mundial 2016-2025}

Parece importante cerrar la presente disertación con el análisis situacional de Cuba forjado a partir de un punto coyuntural y hasta unos años hacia delante. En noviembre de 2016, las noticias políticas de Cuba impactaron al mundo. Ocurrió 
un evento sin precedentes, que implicó la concentración de muchos cubanos, afligidos por la pérdida de su líder. La muerte de F. Castro, dio espacio para monitorear a dónde iría el desarrollo de la política exterior cubana, y que pasaría con su sistema político y social. Sin duda, la clave sociopolítica del Gobierno cubano es una nueva generación de políticos y la creación paralela de nuevas reglas para los temas de la agenda internacional. Es probable que la formación política de Cuba siga el camino de China y Vietnam, donde los líderes carismáticos también gobernaron durante largos años para reemplazar a los grandes líderes comunistas (Fazio Vengoa, 2012; Mingst y Arrguín-Toft, 2018).

Actualmente R. Castro ostenta el rol unificador en Cuba, sin embargo, después de su partida la probabilidad de una lucha de poder entre diferentes frentes podría poner en peligro la supervivencia del Régimen. Y hay razones para creer que esto es a lo que apuestan ciertos círculos en EE. UU. (Sanabia, 2019). Habiendo probado una variedad de estrategias dirigidas al derrocamiento de Castro, cuentan cada vez más con la llamada solución biológica al problema. En su opinión, la muerte de los hermanos Castro, significará automáticamente el fin del Socialismo cubano. Sin embargo, la situación real es mucho más compleja, en una nueva etapa, la unidad se volverá aún más una condición para la supervivencia de la clase dominante cubana (Arias Castaño, 2016; Chomsky, 2016).

Recientemente se han observado nuevas tendencias en el modelo político, ejemplo de ello es el nombramiento de Miguel DíazCanel como presidente del Estado en 2018. El paso por el poder de R. Castro, marcó el inicio del surgimiento de un paulatino proceso de "perfeccionamiento" del socialismo cubano: los residentes adquirieron el derecho a vender y comprar bienes inmuebles y automóviles, a usar internet en sus celulares y a tener un negocio privado. $\mathrm{Y}$ en muchos sentidos, de acuerdo con Berg-Rodríguez (2019), el destino de la República depende de la ejecución exitosa de las reformas que los nuevos líderes estatales parecen entender bastante bien en la actualidad. Desde el 19 de abril de 2018 a hoy, quizá la mayor prueba de liderazgo del actual mandatario, ha sido la celebración de un referendo popular para aprobar una nueva Constitución en la Isla, actuando en un contexto internacional adverso (recrudecido por la hostilidad del Gobierno estadounidense), pero contando especialmente, con el apoyo de sus aliados Rusia y China.

Entre las ventajas económicas se cuenta con que la industria del turismo atrae gran inversión extranjera. La exportación de azúcar, níquel y tabaco está en auge y el sistema bancario se fortalece, pues los bancos están jugando un buen papel en el financiamiento de la economía nacional. Además de ello, destaca la explotación de nuevas reservas de petróleo, puesto que la Isla busca alcanzar autosuficiencia energética en los próximos años. También debe anotarse en este punto el hecho de que, el gobierno aumentó significativamente el número de tipos de actividad empresarial permitidos. En su momento, R. Castro hizo un llamado a los conciudadanos a apoyar su programa radical de cambio económico, diciendo que el futuro de la Revolución pendía de un hilo. Según él, las reformas, implicaban un aumento significativo en el papel del emprendimiento privado para salvar el sistema y no para volver al capitalismo. Entre tanto, la mayor de sus debilidades sigue siendo que a causa del embargo estadounidense, carece de acceso a importantes mercados e inversiones y hay una aguda escasez de moneda (Tamayo, 2015). Pero todo sugiere que mantendrá una amplia agenda internacional y aun con un régimen socialista, continuará la política de poderes blandos aprendida de China.

\section{Conclusiones Finales}

El texto exploró la medida en que los rasgos de personalidad de un tomador de decisiones impactan en sus políticas exteriores. Y si bien las variables idiosincrásicas por sí solas no son los determinantes exclusivos, si tienen un papel importante que desempeñar. La toma de decisiones de política exterior es el resultado de cómo los individuos con poder perciben y analizan los eventos. Y puesto que, a los líderes políticos también les caracterizan los rasgos humanos de subjetividad, prejuicios y sesgos, sus creencias y motivaciones subyacentes influirán en las decisiones a las que lleguen. La cultura, la geografía, la historia, la ideología y las autoconcepciones, analizadas en torno a variables funcionales, burocráticas, nacionales y del contexto internacional, dan forma al proceso de pensamiento de quien desarrolla e implementa la política exterior. 
Cuba, puntualmente, ha creado durante décadas, mecanismos para defenderse contra un embargo económico y la constante presión política e ideológica de EE. UU. y, directa o indirectamente esto ha dado más flexibilidad a sus relaciones económicas y comerciales con el exterior. F. Castro gobernó Cuba desde 1959 hasta 2008 y, gravemente enfermo, cedió el poder a su hermano R. Castro en 2006. Su muerte en 2016 se sintió en Cuba como la desaparición de un poderoso símbolo, cemento de identidad revolucionaria nacional y garante de la ideología del Régimen.

En abril de 2017, R. Castro renunció a sus funciones como Presidente del Consejo de Estado y del Consejo de Ministros, pero conserva el cargo de Primer Secretario del Partido Comunista de Cuba hasta el final de su mandato en 2021. Un nuevo proyecto de constitución, aprobado por la Asamblea Nacional de abril de 2019, reafirma el carácter preeminente del papel del Partido Comunista en la dirección del país y su carácter socialista. La economía cubana sufre el embargo comercial, así como la crisis por la que atraviesa su aliado venezolano y las dificultades económicas y políticas con Brasil. La caída de los precios del petróleo, combinada con graves dificultades financieras, redujo significativamente el flujo de suministros y financiamiento del que se beneficiaba Cuba.

La relación con EE. UU. que ha dado forma a la identidad cubana durante casi 150 años, naturalmente sigue siendo una gran preocupación para el Gobierno cubano (García Sanz, 2011). La administración Trump ha optado por no cuestionar los principales logros obtenidos antes de las elecciones de 2016 (restablecimiento de las relaciones diplomáticas, celebración de diálogos sectoriales regulares y reanudación de los enlaces aéreos). $\mathrm{Y}$ al mismo tiempo, ha tomado varias medidas para castigar y fortalecer las disposiciones del embargo, abriendo incluso la puerta a procedimientos judiciales en los tribunales estadounidenses, contra cualquier persona física o jurídica asociada con una operación de tráfico en relación con bienes nacionalizados por el régimen de Castro desde 1959.

Cuba también, se enfrenta a un entorno regional cambiante, con la desaparición de sus aliados regionales (especialmente Brasil de Lula/ Rousseff) y las graves dificultades de su apoyo
(Venezuela, Nicaragua). Por lo cual, busca nuevos socios: en ALC, especialmente en forma de alianzas multilaterales; en Asia donde encuentra fuentes de inspiración y; finalmente en Europa, en donde aún todo se queda en intenciones, pues, sus dificultades políticas internas no le han permitido establecer una relación tan estrecha. Sin embargo, entre los países europeos significativamente presentes (Francia, Italia, Alemania y los Países Bajos) en Cuba, estos lo hacen con algunas pequeñas inversiones. Pero aun cuando la Unión Europea y Cuba firmaron el 12 de diciembre de 2016, en Bruselas, un acuerdo de cooperación y diálogo político que cubre todos los temas de interés común: derechos humanos, lucha contra la corrupción, buen gobierno y cambio climático. Con todo, aún no se ve mucho avance en la materia.

Los dos principales socios fuera de la región son Rusia, que ha decidido hacer de Cuba uno de sus puntos de anclaje en la región, intensificando una vez más las relaciones y proyectos, desde la cancelación y reprogramación de la deuda de la era soviética. Y China, que se ha convertido en su principal socio comercial. Y al final, todo parece indicar que, Cuba mantendrá un régimen socialista, continuará con la tradicional política y con un desarrollo socioeconómico, basado en la experiencia de China. De manera que Cuba tiene un gran potencial en las relaciones con sus socios asiáticos, pero hay un largo camino por recorrer, así que, todo dependerá del ritmo de las reformas económicas y la voluntad política, tanto de Cuba como de los demás países para aprovechar la oportunidad.

\section{Referencias}

Alburquerque, G., y Alvarado, D. H. (2019). Cuba, ¿un obstáculo a la participación latinoamericana en el Movimiento de Países No Alineados? (19611984). Autoctonía. Revista de Ciencias Sociales e Historia, 3(1), 54-67. doi: 10.23854/autoc.v3i1.111

Alzugaray, C. (2014). La actualización de la política exterior cubana. Política Exterior, 28(161), 70-82.

Arias Castaño, L. (2016). La actualización del modelo socialista y sus efectos en el Partido Comunista Cubano (Doctoral dissertation, Universidad del Rosario). 
Ayerbe, L. F. (2016). Los impactos del restablecimiento de relaciones entre Cuba y EE. UU. en América Latina y el Caribe. Contextualizaciones Latinoamericanas, (15).

Banderas Bustillos, S. E. (2016). Análisis de la continuidad del embargo impuesto por EE. UU. a Cuba (2001-2013) (Bachelor's thesis, PUCE).

Berg-Rodríguez, A. (2019). La reforma constitucional en Cuba en el marco de la aplicación provisional del Acuerdo UECuba del 2016 (The Constitutional Reform in Cuba in the Context of the Provisional Implementation of the 2016 EU-Cuba Agreement). Oñati Socio-Legal Series, 9(6), 924-950. doi: 10.35295/osls.iisl/0000-00000000-1109

Bobes, V. C., Mesa-Lago, C., Chaguaceda, A., Geoffray, M. L., Alfonso, H. D., y Rojas, R. (2015). Cuba:¿ Ajuste o transición? Impacto de la reforma en el contexto del restablecimiento de las relaciones con EE. UU.. FLACSO Mexico.

Bueno, S. (2015). Medio siglo de literatura cubana. Revista de la Biblioteca Nacional José Martí, (4).

Caetano, G., y Sanahuja, J. A. (2019). Integración regional y regionalismo en crisis. Revista uruguaya de Ciencia Política, v. 28, n. 1, pp. 7-14.

Campos, C. O. (2011). Las relaciones de Cuba con América Latina y el Caribe: Imperativos para consolidar la reinserción regional. Cuba, EE. UU. y América Latina frente a los desafios hemisféricos.

Cavicchioli, M. I. (2014). La doctrina neoconservadora y el excepcionalismo americano: Una vía al unilateralismo ya la negación del Derecho Internacional. Revista electrónica de estudios internacionales (REEI), (28), 5-36.

Chaveco, E. N. P., y del Río, J. A. O. (2010). Retos y perspectivas del sistema político cubano. Universidad de Holguín” Oscar Lucero Moya”.

Chomsky, N. (2016). Hegemonía o supervivencia: la estrategia imperialista de EE. UU. Barcelona: Ediciones B.
Correa Serrano, M. A., y Catalán Salgado, E. (2016). La Alianza del Pacífico: Entre la geopolítica de China y de EE. UU.. México y la Cuenca del Pacífico, 5(14), 19-52. doi: 10.32870/mycp.v5i14.505

Domínguez Guadarrama, R. (2015). Cuba y EE. UU.: el largo proceso del reconocimiento. Latinoamérica. Revista de Estudios Latinoamericanos, (60), 53-92. doi: 10.1016/j.larev.2015.04.001

Duany, J., Ángel, S., Liendo, N. A., González, C., Bobes, C., Chaguaceda, A., y SalazarElena, R. (2019). Cuba pos-Castro ¿espejismo o realidad?: miradas diversas sobre una sociedad en transición. Bogotá: Universidad Sergio Arboleda.

Edwards, I. J. (2015). La seguridad de Cuba y los noventa. Sociológica México, (25).

Fazio Vengoa, H. (2012). Rusia y China: de la disimilitud comunista a la divergencia poscomunista: contraste de experiencias. Ediciones Uniandes-Universidad de los Andes.

Feinberg, R. (2011). Extender la mano: La nueva economía de Cuba y la respuesta internacional. Iniciativa para las Américas. Instituto Brookings.

Flores, M. J. A. (2018). La relación bilateral Cuba-EUA: del bloqueo económico a la distensión (2014-2016). (Bachelor's thesis, El Colegio de San Luis)

García Sanz, M. D. (2011). Crisis de la hegemonía estadounidense: Análisis comparativo de las posturas de Zbigniew Brzezinski e Immanuel Wallerstein (Bachelor's thesis, QUITO/ PUCE/2011).

Gómez, D. A. (2013). China y su relación con América Latina. Una aproximación desde el poder blando1. Relaciones Políticas e Internacionales, 47.

Gómez, J. G. (2015). La Cuestión de Cuba en 1884. Revista de la Biblioteca Nacional José Martí, (1).

Hernández, R., Domínguez, J. I., y Schultz, R. (2013). Cuba, la actualización del modelo. Balance y perspectiva de la transición socialista. Harvard: Harvard University/Temas. 
Hornero, A. C. (2016). Economía internacional y organismos económicos internacionales. Editorial Centro de Estudios Ramón Areces S.A.

Iglesias, M. (2002). Los sucesos del $11 \mathrm{de}$ septiembre y después. ¿ Choque de civilizaciones?. "Por la construcción de una verdadera comunidad internacional". La Plata, 14 y 15 de noviembre.

Izuel, E. B., Cervera, R. C., Prieto, N. C., Segura, C. G., Hernández, R. G., Muñoz, J. I., ... y Gatica, J. P. S. (2015). Teorías de las relaciones internacionales. Tecnos.

i Lecha, E. S. (2011). La UE y Turquía: la adaptación a un mundo multipolar. Cuadernos de estrategia, (151), 253-288.

Jeifets, V., y Jeifets, L. (2017). El encuentro de la izquierda cubana con la Revolución Rusa: el Partido Comunista y la Comintern. Historia crítica, (64), 81-100. doi: 10.7440/histcrit64.2017.05

Kuwayama, M., y Rosales, V. (2012). China y América Latina y el Caribe: Hacia una relación económica y comercial estratégica. CEPAL.

León, M. M. U. (2012). ¿Hacia una civilización universal? Una crítica del «choque de civilizaciones» de Huntington. Estudios de Deusto, 56(2), 105-133. doi: 10.18543/ed56(2)-2008 pp105-133

López Zea, L., y Zea Prado, I. (2010). Los tres pilares de Rusia en América Latina (después de la Guerra Fría). Revista de Relaciones Internacionales de la UNAM, (108).

Llenderrozas, E. (2013). Política exterior latinoamericana y la Comunidad de Estados Latinoamericanos y Caribeños. Desafíos estratégicos del regionalismo contemporáneo: CELAC e Iberoamérica, 129.

Malamud, C. (2011). América Latina frente al conflicto libio: respuestas a la Resolución 1973. Boletín Elcano, (135), 8.

Márquez Covarrubias, H. (2010). Crisis del sistema capitalista mundial: paradojas y respuestas. Polis. Revista Latinoamericana, (27). doi: 10.4067/S071865682010000300020
Mingst, K. A., y Arrguín-Toft, I. M.

(2018). Fundamentos de las relaciones internacionales. CIDE.

Natanson, J. (2012). La nueva izquierda: Triunfos y derrotas de los gobiernos de Argentina, Brasil, Bolivia, Venezuela, $C$. Debate.

Panitch, L., y Gindin, S. (2015). La construcción del capitalismo global: la economía política del imperio estadounidense (Vol. 84). Ediciones Akal.

Perea Ozerin, I. (2014). Incidencia de las Revoluciones en los Movimientos Sociales Transnacionales. El caso de la Revolución Cubana y su influencia en el Movimiento Antiglobalización. (Doctoral dissertation, Universidad del País Vasco, Euskal Herriko Unibertsitatea)

Ríos, X. (2016). China-América Latina y Caribe: otra relación para otro futuro. Pensamiento Propio, (44), 217-247.

Rodrigo, Á. J. (2016). Entre Westfalia y

Worldfalia: La comunidad internacional como comunidad social, política y jurídica. En: La tensión cosmopolita. Avances y límites en la institucionalización del cosmopolitismo, Madrid, Tecnos, 23-63.

Rodríguez, J. L. (2011). Cuba, su economía y la Unión Soviética. Temas, 68(3), 1-17.

Sanabia, D. (2019). Trump contra Cuba y Venezuela. Semanario Internacional de Prensa Latina, Agencia Informativa Latinoamericana S.A. año III No.19 semana del 8 al 14 de mayo de 2019

Santa Cruz, A. (Ed.). (2014). El constructivismo y las relaciones internacionales (Vol. 1). CIDE.

Santos Brito, L. P. (2019). ¿Es la Federación Rusa una gran potencia hoy en día? (Bachelor's thesis, Quito: Universidad de las Américas, 2019).

Sarsenov, M. B., y Raev, D. S. (2018). La humanización como fenómeno del sistema de relaciones internacionales post-bipolar. Utopía y Praxis Latinoamericana, 23(82), 136-146. 
Serbin, A. (2011). Círculos concéntricos: la política exterior de Cuba en un mundo multipolar y el proceso de 'actualización'. Cuba, EE. UU. y América Latina frente a los desafios hemisféricos. Buenos Aires: CRIES/Icaria Editorial.

Serbin, A. (2015). ¿Un nuevo ciclo del regionalismo latinoamericano en el siglo XXI? Desafíos y limitaciones después de la normalización de las relaciones entre Cuba y EE. UU.

Serbin, A. (2016). ¿Fin de ciclo?:

Las relaciones entre Cuba y EE. UU. en el entorno regional y global. En: ¿Fin de ciclo y reconfiguración regional? América Latina y las relaciones entre Cuba y EE. UU.

Silva, M. R. (2014). La hora de los desconectados: evaluación del diseño de la política de "acceso social" a internet en Cuba en un contexto de cambios. Crítica Y Emancipación, VI, 11, 291-377.

Stoessel, S. (2014). Giro a la izquierda en la América Latina del siglo XXI: Revisitando los debates académicos. Polis (Santiago), 13(39), 123-149. doi: 10.4067/ S0718-65682014000300007
Tamayo, M. D. J. V. (2015). La confianza política en el pensamiento político de F. Castro. Una aproximación. Estudios del Desarrollo Social: Cuba y América Latina, 4(1), 138-159.

Vélez Pretelt, L. A. (2011). Análisis de la autonomía de la política exterior cubana frente a su dependencia económica de la Unión Soviética en la década de los sesenta (Doctoral dissertation, Universidad del Rosario).

Velez Vargas, J. C. (2015). Rusia, un reto al orden mundial posterior a la guerra fría: política exterior rusa y su rol en la configuración del sistema internacional del siglo XXI. (Master's thesis, Pontificia Universidad Javeriana).

Zovatto, D. (2014). El estado de las democracias en América Latina. Perú: JNE y EEG, Serie Cátedra Democracia N. ำ1.

Zúñiga, D. L. (2012). Repaso de las relaciones económicas internacionales de la revolución cubana: Una historia de conflicto y cooperación. Relaciones Internacionales, 83. 